\section{A macromolecular build-up}

\author{
C.D. Rodger
}

Molecular Structure: Macromolecules in Three Dimensions. By Robert J. Fletterick, Trina Schroer and Raymond J. Matela. Blackwell Scientific:1985. Pp.196. Pbk £19.80, \$19.95.

THE greater availability these days of molecular model-building kits has encouraged many of us teaching undergraduates in biology and biochemistry to supplement the theoretical material with practical "molecular construction" exercises. Molecular Structure is aimed at those using this approach. The book is unusual, however, in that it is attempting to be two things in one - a handbook for use with the molecular model-building kit also produced by Blackwell, and an explanatory textbook in its own right. Book and modelling kit can be purchased separately, though it is difficult to review the book without including some reference to the models as well.

The text is divided into three parts. The first ("Molecules and Modelling") contains a general introduction to molecular symmetry and the Blackwell modelling

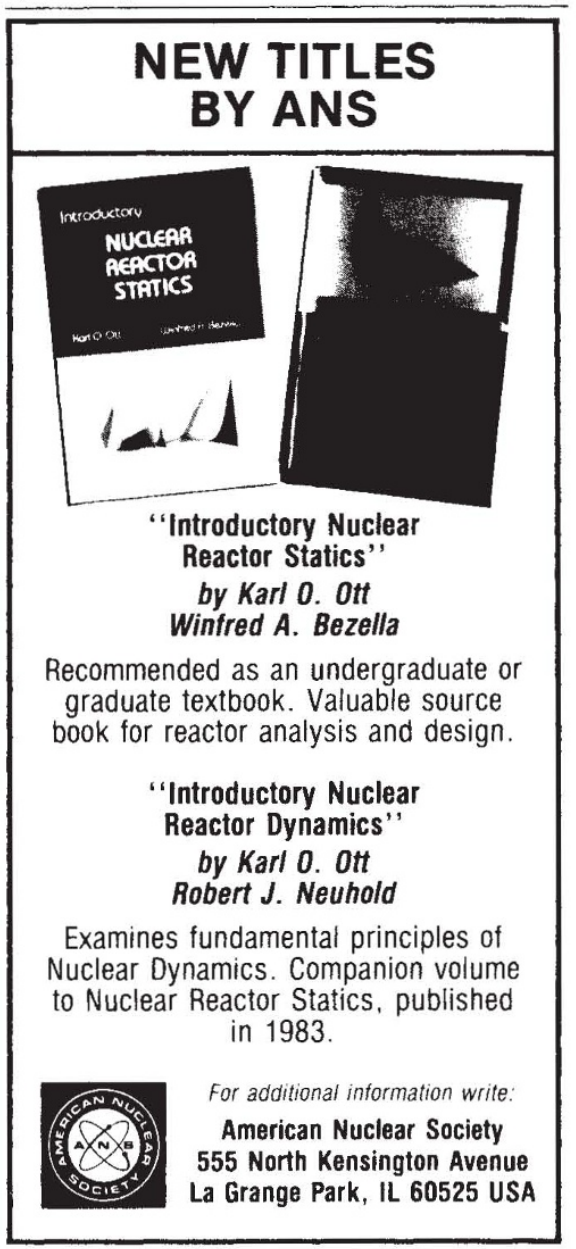

Reader Service No.4 system. The Blackwell models are not of the "all-atom" type and are intended for the construction of the backbones of proteins and nucleic acids, but not the side chains. They enable particularly accurate setting of the backbone angles because a small vernier is built into each of the components, thus allowing the angles to be set to an accuracy of $1^{\circ}$. Despite this precision, it seems that all the exercises in the book can be accomplished in a single practical session.

Part II, "Protein and Nucleic Acid Structures", introduces the concepts of folding and domains in polypeptides, and then continues with more detailed accounts of the secondary structures of polypeptides, together with instructions for constructing them from the kits. The same format is then used for describing nucleic acid structures. The descriptions are quite thorough, but the resulting backbone models of secondary structures seem rather crude by comparison. I suspect many would not consider the time spent building these particular structures as backbone models to be worthwhile at undergraduate level; such models cannot demonstrate useful concepts, such as the dominance of side chains in the $\alpha$-helix or the relative tilts of bases in different nucleic acids, as well as all-atom kits. The backbone kits come into their own, however, when the book explains the construction of transfer RNA at the end of this section; definitely not a task for the all-atom approach in a student practical!

\section{Biology's conscience}

\section{E.G. Richards}

Physical Chemistry: Principles and Applications in Biological Sciences, 2nd Edn. By Ignacio Tinoco, Jr, Kenneth Sauer and James C. Wang. Prentice-Hall:1985. Pp.706. \$38.95, £41.70.

Physical chemistry, it has been said, is the conscience of the biologist. We expect the mechanisms propounded by biochemists and molecular biologists to conform to the laws of physics, and because we are dealing with molecules, physical chemistry is the bridge. Even if one does not take this reductionist position, it must be admitted that the subject is at least the hand-maiden of the modern biology which would be infinitely poorer were it not for such experimental techniques as ultracentrifugation, electrophoresis or X-ray crystallography.

In the new edition of their book, Tinoco, Sauer and Wang, all expert practitioners of physical chemistry, provide the foundation for a sound understanding of the subject, as well as insights into some of the important techniques. The book is
Part III contains instructions for building more complex structures such as insulin, the cro repressor and lysozyme. Although these are more complicated molecules, construction time is still within three hours because they are backbone models. It is with these structures that the backbone kits are likely to be most useful.

At first glance it appears that saccharide conformations are ignored altogether. However some details are included in Part III, in the instructions for building the lysozyme substrate containing $\mathrm{N}$-acetylmuramic acid and $\mathrm{N}$-acetylglucosamine. The authors have recognized that the backbone type of molecular modelling is unsuitable for demonstrating the intimacy of active-site substrate complexes and they include guidance on the use of LabQuip model kits for this exercise.

The text is well set out and easy to read. It contains many illustrations, albeit with rather sparing use of colour, and references are included at the end of each chapter. Representing three-dimensional structures on the flat page is not easy, and I suspect that some of the illustrations may not in fact help the reader to visualize the three-dimensional arrangements as the authors intended. Nevertheless, in its role as a companion handbook to a modelbuilding kit the book should prove very useful.

C.D. Rodger is Biology Course Leader in the Department of Sciences, Roehampton Institute of Higher Education, London SWI5 5 PJ, UK. specially written for the biologist, by which the authors mean the biochemist, biophysicist and molecular biologist; as such it invites comparison with Cantor and Shimmel's three-volume Biophysical Chemistry, but it is more appropriate for the beginner and thus fills an important gap. The coverage of fundamentals is good and geared to the interests of the intended audience. Some of the more esoteric methods, however, such as inelastic light scattering, are discussed but briefly with mere quotation of the relevant formulae.

One invaluable feature is the large number of worked examples in the text and of problems for the student (about one-third with answers at the back). Another, at any rate of the first half of the book, is the absence of higher mathematics, though more mathematical expertise is required for the later chapters on X-ray diffraction and quantum mechanics. The second edition has been improved by the near universal use of SI units, as well as the inclusion of extra topics. Altogether, it should remain a very popular textbook.

E.G. Richards is a part-time lecturer in the Department of Biophysics, Cell and Molecular Biology, King's College London (KQC), 26-29 Drury Lane, London WC2B 5RL, UK. 